\title{
Gastrointestinal Calcinosis as a Rare Cause of Death in Juvenile Polymyositis
}

\author{
Maryam Broomand ${ }^{1}$, Vahid Ziaee ${ }^{1,2,3}$, Mohammad-Hassan Moradinejad ${ }^{1,3}$ and Hosein Alimadadi ${ }^{1,3,{ }^{*}}$ \\ ${ }^{1}$ Children's Medical Center, Pediatrics Center of Excellence, Tehran, Iran \\ ${ }^{2}$ Pediatric Rheumatology Research Group, Rheumatology Research Center, Tehran University of Medical Sciences, Tehran, Iran \\ ${ }^{3}$ Department of Pediatrics, Tehran University of Medical Sciences, Tehran, Iran \\ "Corresponding author: Children’s Medical Center, No. 62, Dr. Gharib St., Keshavarz Blvd., Tehran, Iran. Email: hoseinalimadadi@yahoo.com
}

Received 2017 November 11; Revised 2018 August 12; Accepted 2018 August 17.

Keywords: Dermatomyositis, Polymyositis, Calcinosis, Gastrointestinal Involvement, Mortality

\section{Dear Editor,}

Juvenile polymyositis (JPM) (or Dermatomyositis) is a rare autoimmune vasculopathy of childhood that preferentially affects dermal and muscular vessels (1). Ectopic calcification has been reported as a complication of JPM. It can cause significant debility with severe pain, skin ulceration, muscle atrophy, joint contracture, and acroosteolysis (2). Although early diagnosis and treatment with immunosuppressive drugs may decrease this complication, there is no established medical treatment (1-3).

A 9 years old boy had referred to our hospital with muscle weakness and increase of CPK and aldolase without any skin signs. He was treated with the diagnosis of post viral myositis and discharged. But he returned some weeks later with intensification of muscle weakness. Corticosteroids were started with the diagnosis of polymyositis.

Two years later, when the disease was in remission, we visited him for acute abdominal pain. Appendicitis was diagnosed and appendectomy reveled a perforated appendicitis. Again two years later he came with gastrointestinal (GI) manifestations such as vomiting and GI bleeding. He had fever, malaise and abdominal pain. He was admitted with primary diagnosis of partial obstruction. He was ill and febrile and had tachycardia and hypotension. His abdomen was distended, tender and had no defecation for several days. As paralytic ileus due to electrolyte disturbance and septic shock, he was treated with antibiotics, and electrolyte imbalance was corrected. Double contrast abdominal CT scan showed bowel wall thickening due to calcinosis (Figure 1). Because of not responding to conservative treatment necrotic bowel was resected and ileostomy fixed. In macroscopic view, total bowel was edematous, dilated and calcified. Ten days after operation the patient was again febrile and had pus discharge from the site of incision and 5 days later had respiratory distress and $\mathrm{O}_{2}$ saturation was decreased. He had to be intubated. Chest $\mathrm{X}$ ray showed consolidation, pneumatocele and calcinosis universalis. Unfortunately 3 days later he died due to cardiopulmonary arrest following the universal calcinosis of the chest and abdomen.

Calcinosis is the hallmark sequel of JPM, occurring mainly in pediatric patients and may occur in muscles, skin, subcutaneous tissue and fascial planes. In majority of the cases, calcinosis develops in the first three years after disease onset, as in our case. Calcinosis occurs in the presence of normal metabolism in damaged or devitalized tissues, is widespread in connective tissue diseases with a prevalence of $30 \%$ to $70 \%$ in JPM $(3,4)$.

The mechanism of calcinosis genesis in JPM is unknown but it may be explained by a dystrophic mechanism with release of mitochondrial calcium from damaged muscle into matrix vesicles. Hydroxyapatite has been seen in histological study of the calcinosis (5).

In patients with GI disease, dysphagia and bowel motility disorder were the most common complaints. One patient with GI disease underwent surgery for massive hemorrhagic perforated duodenal ulcer (6). Large calcium deposits may be the source of considerable morbidity and functional disability. For palliation, complete surgical excision of larger lesions may be the treatment of choice (4). Our patient had vomiting, abdominal pain and no defecation. With the primary diagnosis of partial obstruction laparotomy was performed. The bowel was totally dilated and calcified.

Risk factors for developing calcinosis in patients with JPM are not well defined. The calcinosis is associated with 

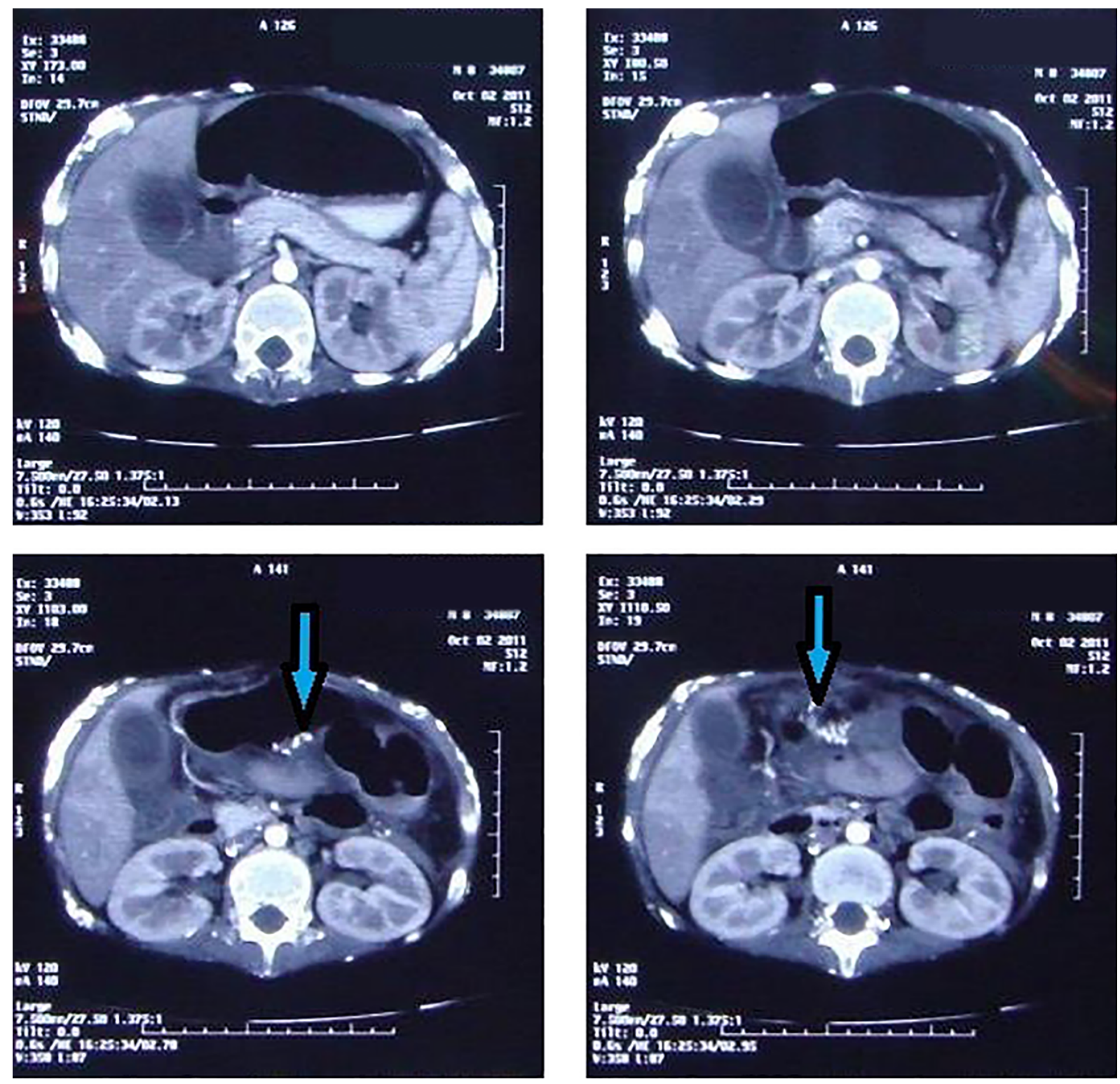

Figure 1. Calcinosis in the bowel wall of the patient with juvenile polymyositis

delay in diagnosis and initiation of therapy, chronic severe disease and inadequate therapy $(2,3)$. There is no universally recognized treatment for calcinosis associated with JPM. Multiple treatment strategies (aluminum hydroxide, diltiazem, probenecid, bisphosphonates, colchicine, local triamcinolone and infliximab) have been used to treat calcinosis with variable success (4). In patients with refractory muscle weakness or lethal complications, intravenous immunoglobulin was added to the treatment regimen as in our patient (7).
Lung diseases such as pneumonia and interstitial lung disease as well as GI involvement like GI vasculitis and GI muscle weakness are the main causes of mortality in the acute phase of the disease $(8,9)$, but universal calcinosis of bowel and lungs itself can lead to death.

Although calcinosis is a common complication of JPM, mortality due to this complication is very rare. Calcinosis of pulmonary and GI system should be considered in patients with GI or pulmonary symptoms in the late stage of JPM. 


\section{Footnotes}

Conflict of Interests: The authors declared that they have no conflict of interests.

Funding/Support: It is not declared by the authors.

\section{References}

1. Pachman LM. Juvenile dermatomyositis. Pathophysiology and disease expression. Pediatr Clin North Am. 1995;42(5):1071-98. doi: 10.1016/S0031-3955(16)40054-4. [PubMed: 7567187].

2. Saini I, Kalaivani M, Kabra SK. Calcinosis in juvenile dermatomyositis: Frequency, risk factors and outcome. Rheumatol Int. 2016;36(7):961-5. doi: 10.1007/s00296-016-3467-6. [PubMed: 27007612].

3. Malek A, Raeeskarami SR, Ziaee V, Aghighi Y, Moradinejad MH. Clinical course and outcomes of Iranian children with juvenile dermatomyositis and polymyositis. Clin Rheumatol.2014;33(8):1113-8. doi: 10.1007/s10067-014-2675-2. [PubMed: 24878926].

4. Boulman N, Slobodin G, Rozenbaum M, Rosner I. Calcinosis in rheumatic diseases. Semin Arthritis Rheum. 2005;34(6):805-12. doi: 10.1016/j.semarthrit.2005.01.016. [PubMed:15942915].
5. Anderson HC, Morris DC. Mineralization. In: Barrett JE, editor. Handbook of experimental pharmacology. 17. Heidelberg: Springer-Verlag; 1993. p. 267-98.

6. Chowdary N, Benakappa A, Dakshayani RM. Juvenile Dermatomyositis in an Indian girl: A rare condition in childhood - A case report. British Biomedical Bulletin. 2015;3(3):299-303.

7. Marie I, Menard JF, Hatron PY, Hachulla E, Mouthon L, Tiev K, et al. Intravenous immunoglobulins for steroid-refractory esophageal involvement related to polymyositis and dermatomyositis: A series of 73 patients. Arthritis Care Res (Hoboken). 2010;62(12):1748-55. doi: 10.1002/acr.20325. [PubMed: 20722047].

8. Santo AH, Souza JM, Pinheiro CE, Souza DC, Sato EI. Trends in dermatomyositis- and polymyositis-related mortality in the state of Sao Paulo, Brazil, 1985-2007: Multiple cause-of-death analysis. BMC Public Health. 2010;10:597. doi: 10.1186/1471-2458-10-597. [PubMed: 20937106]. [PubMed Central: PMC3091559].

9. Singh S, Suri D, Aulakh R, Gupta A, Rawat A, Kumar RM. Mortality in children with juvenile dermatomyositis: Two decades of experience from a single tertiary care centre in North India. Clin Rheumatol. 2014;33(11):1675-9. doi: 10.1007/s10067-014-2747-3. [PubMed: 25053380]. 